\title{
Epilepsy in a case with bilateral hippocampal sclerosis
}

\author{
Cristina Caciuc ${ }^{1}$, Razvan Motoc ${ }^{1}$, Amalia Ghergu ${ }^{1}$, Alexandra Oprisan ${ }^{1,2}$ \\ ${ }^{1}$ Department of Neurology, Colentina Clinical Hospital, Bucharest, Romania \\ 2Department of Neurology, "Carol Davila" University of Medicine and Pharmacy, Bucharest, Romania
}

\begin{abstract}
Mesial temporal sclerosis (MTS) is a pathology usually seen in young individuals. It is responsible for partial seizures, with possible secondary generalization. It may be initially managed with antiepileptic agents, but eventually some patients may be refractory to medical treatment. Authors report the case of a 21-year-old man with generalized tonic-clonic seizure and a suggestive cerebral MRI for bilateral hippocampal sclerosis. MTS remains a controversial pathology, which requires a long term management, with an unpredictable evolution.
\end{abstract}

Keywords: mesial temporal lobe epilepsy, hippocampal sclerosis, generalized tonic-clonic seizures, memory impairment

\section{INTRODUCTION}

Mesial temporal sclerosis is responsible for about $60 \%$ of the temporal lobe epilepsies (1). It associates unprovoked seizures with onset in the mesial or limbic structures of the temporal lobe and hippocampal sclerosis (2). Its etiology is unknown and controversial. It is considered an acquired pathology, but familial cases have been documented $(1,3)$. Febrile convulsions early in life, genetic susceptibility, inflammation, neurodevelopmental factors are seen as possible mechanisms through which the epileptogenic focus occurs (1,3). Mesial temporal sclerosis is responsible for partial seizures, with possible secondary generalization (1). Mesial temporal lobe epilepsy (MTLE) is initially managed with antiepileptic agents (3). Most patients are refractory to medical treatment; thus, the anterior temporal lobectomy or selective amygdalo-hippocampectomy is a therapeutic management for these patients (2).

\section{CASE STUDY}

We present the case of a 21 year old boy, with a history of febrile seizures at the age of three, with- out other significant medical history, who had a generalized tonic-clonic seizure one week before admission in our hospital. He was found by his family with altered consciousness and abnormal breathing. He had no traumatic head signs or traumatic tongue injury, and no loss of sphincter control. Time of onset and the duration of seizure were uncertain. He was hospitalized and monitored for 24 hours. On discharge, his family noticed memory impairment and disorientation, which persisted in the following days.

On admission in our clinic eight days later, general examination was normal. The neurologic exam revealed a conscious, vigilant patient, without signs of meningeal irritation, without signs of cranial nerves pathology, no motor deficit, with symmetrical deep tendon reflexes, with plantar cutaneous reflex in flexion. The patient had no superficial or profound sensitivity disturbances. He had a MMSE (mini mental state examination) of $25 / 30$ with short-term memory loss, retrograde and anterograde memory impairment.

Laboratory blood evaluation revealed normal cell and platelet counts, without inflammatory syndrome. Biochemical laboratory serum values, coagulation parameters were within the normal range. 
The possibility of an infectious process was considered and a serological screen for syphilis and HIV (human immunodeficiency virus) was conducted, with negative results.

An EEG was performed - it did not reveal any pathological discharges.

The next step was to perform a lumbar puncture, which revealed normal appearance and normal opening pressure of the cerebrospinal fluid (CSF), negative Pandy's test, normal white blood count ( 1 cell/uL), normal glucose and protein concentrations. His CSF tested negative for herpex simplex virus type 1, 2 (HSV1,2) - DNA and varicella-zoster virus $(\mathrm{VZV})-\mathrm{DNA}$.

Brain magnetic resonance T2-weighted imaging and FLAIR (1,5 Tesla) revealed an increased hippocampal signal, with hippocampal decreased volume, affecting both mesial temporal lobe structures. Also, other findings included: temporal lobe volume loss, lack of contrast enhancement.

A magnetic resonance spectroscopy (MRS) of bilateral hippocampus was performed. It detected a slightly increased in choline and creatine levels, with no significant difference in both mesial temporal lobes, with no change in Cho/NAA, Cho/Cr ratios.

Considering the medical history, the age, the clinical examination and the final results of the tests performed, the patient was diagnosed with bilateral mesial temporal sclerosis. We identified a possible trigger factor - febrile seizures in childhood. Treatment with antiepileptic drug was initiated - sodium valproate $750 \mathrm{mg}$ daily. His evolution during hospitalization was favorable, without any new seizures, and he had better results in memory tests. At 2 weeks follow-up, his liver function tests were high ( $>3 \mathrm{x}$ normal values), so his current neurologist decided to replace valproic acid with levetiracetam $1,000 \mathrm{mg} /$ daily. At 3 months follow-up - he remained seizure free, with no memory impairment.

\section{DISCUSSION}

Hippocampal sclerosis was first identified in 1825 by Bouchet and Cazauvieilh, during postmortem examination (1). It is associated with the syndrome of MTLE which was included in the classification of the International League Against Epilepsy (ILAE) in 1989 under the group of localization-related symptomatic epilepsies (4).

In large epilepsy surgical series, MTLE incidence varies between $33.6-66.0 \%$ and in postmortem series it is identified in $30.5-45.0 \%$ of all epilepsy syndromes (1). The temporal lobe epilepsy with mesial temporal sclerosis (MTS) is usually seen in young patients - it can be present from infancy to thirties $(3,5)$. The etiology of hippocampal sclerosis (HS) remains unknown, with possible in-
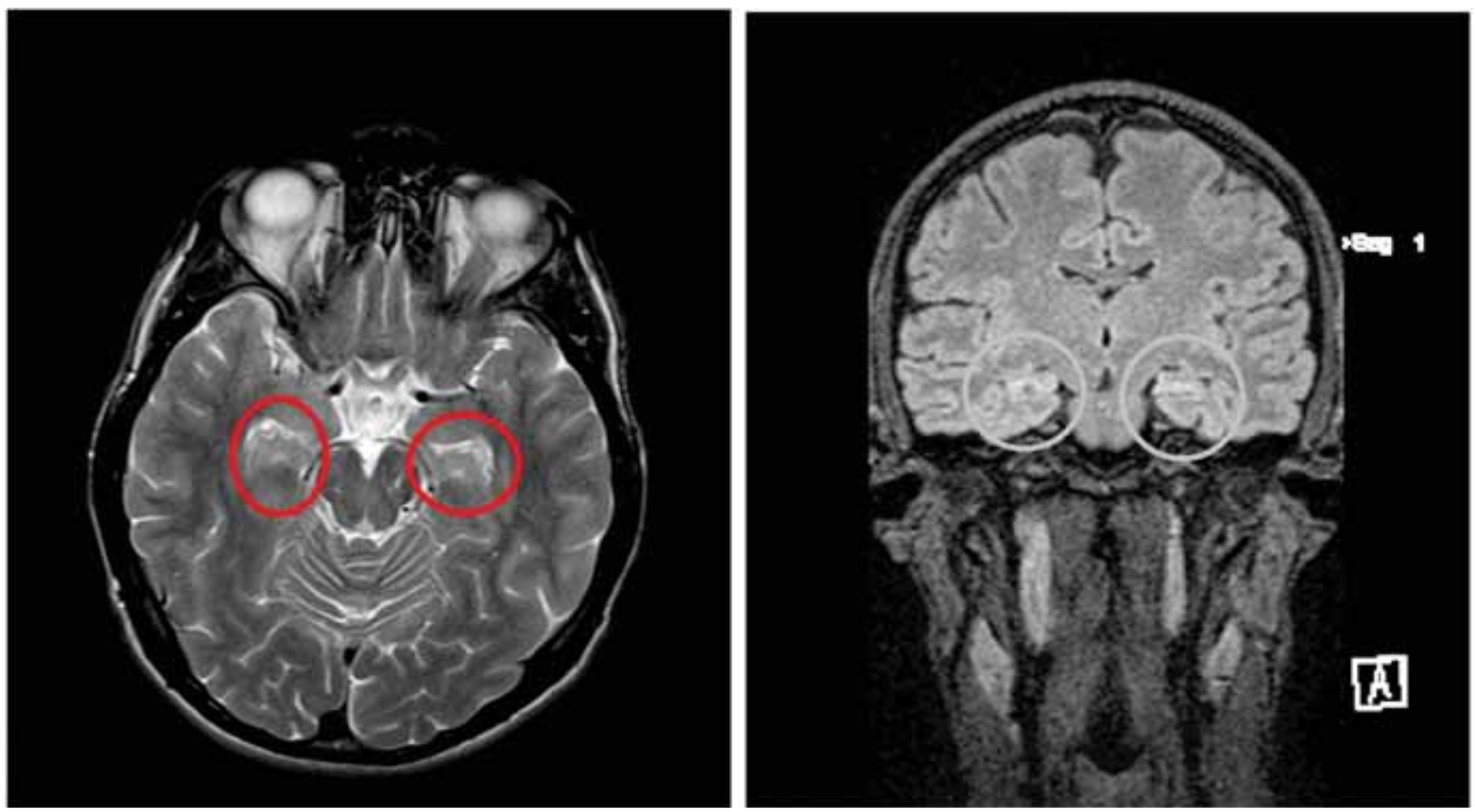

FIGURE 1. Axial T2W and FLAIR coronal images showing asymmetrical T2, FLAIR hyperintense lesions in bilateral mesial temporal lobe structures with hippocampal atrophy. 
volvement of febrile seizures, genetic susceptibility, inflammatory and neurodevelopmental factors (1). Retrospective studies from surgical series have demonstrated a high incidence of "initial precipitating incidents": febrile seizures, trauma, hypoxia and intracranial infections, usually before 5 year old $(3,6)$. These patients classically have a latent period between the trigger factor and onset of seizure (1). Whether the febrile seizures are associated with MTS is controversial - up to $1 / 3$ of patients with MTLE have a history of febrile seizures and follow up of children with febrile seizure did not demonstrate an increased prevalence of MTLE $(7,8)$.

From a neuropathological point of view, hippocampal sclerosis refers to neuronal loss and gliosis in hippocampal and extrahippocampal tissues, such as the amygdala and parahippocampal gyrus (1-3). Several patterns of HS have been recognized, atypical patterns of HS being associated with poorer surgical outcomes (9). These subtypes of HS may point to distinct syndromes with unique pathophysiology $(1,9)$. In the context of hippocampal neuronal loss, seizure-induced hippocampal changes are seen: granule cell dispersion, axonal sprouting are hallmark pathological features of hippocampal sclerosis $(1,2,10)$. In addition, alterations of interneuronal populations represent adaptive or compensatory response to seizure, but may contribute in the pro-epileptogenic process $(1,2)$. Smaller hippocampal volumes than normal are at risk for hippocampal sclerosis (1). In postmortem studies, atrophy outside of mesial temporal structures has been noted, presumably involving hippocampal projection pathways - ipsilateral mammillary body, fornix, thalamus, cingulate, frontal and temporal neocortex (9). Hippocampal sclerosis can be observed in the association with malformations of cortical development (dual pathology) and with focal cortical dysplasia - the role of these abnormalities (epiphenomenon or active participant) is still uncertain $(1,9)$.

MTLE has an onset with partial awareness, with auras, motor arrest, automatisms and autonomic changes $(3,11)$. An interesting aspect in this case was with generalized seizure at the onset. But in most of these cases, it may be a partial temporal seizure with secondary generalization (3). Secondarily generalized seizures may correlate with lesion extension $(3,5)$.

The persistence of memory impairment after 14 days and the progressive recovery may be correlated with a long, severe seizure and the lesion extension. In time, progressive behavioral changes can occur, and also increasing memory impairments $(3,6,12)$. The degree of memory impairment correlates with the degree of atrophy, age of seizure onset, frequency and severity of seizures and additional lesions acquired $(3,16)$.

Interictal EEG may be normal. Usually, electrodiagnostic features in MTLE include interictal anterior or mid-temporal spikes ipsilaterally to the focus (3). Nevertheless, several studies showed that epileptiform activity originated in mesial temporal region is often silent to standard scalp-EEG electrodes (13), and special intracranial techniques are often necessary (14). Although a hippocampal discharge might have no detectable scalp-EEG correlates, relatively typical clinical signs and symptoms, together with typical MRI findings, can be diagnostic $(3,14)$.

The patient's brain MRI was suggestive of bilateral mesial temporal sclerosis. HS is bilateral, usually asymmetrical in about $50 \%$ of epilepsy in postmortem studies (9). On MRI, the hallmark lesion of MTS is the presence of hippocampal sclerosis, demonstrable on coronal MRI sequences by decreased, asymmetrical hippocampal volume, decreased signal intensity seen on $\mathrm{T} 1$ and increased signal on T2-weighted, FLAIR sequences $(3,9)$. These findings, atrophy and hyperintensity are known as the two primary MRI findings of MTS $(3,6)$. Other findings are: loss of the normal internal architecture of the hippocampus, temporal lobe volume loss, enlargement of the temporal horn, narrowed collateral white matter, smaller fornix, an atrophic mammillary body and also blurring at the grey-white matter boundary, usually ipsilateral to the sclerotic hippocampus - these findings can help in the lateralization of MTS and determine which side to resect (15).

Magnetic resonance spectroscopy (MRS) in MTS usually detects decreased NAA and decreased NAA/Cho, NAA/Cr ratios, and either normal and increased $\mathrm{Cho} / \mathrm{Cr}$ ratio $(3,17)$. The metabolite changes seen in MRS often precede structural changes seen by MRI (17). In the presented case, MRS did not identify any pathological changes.

Regarding medical treatment with antiepileptic drugs, $1 / 3$ of patients are seizure-free on medication alone, whereas nearly $2 / 3$ of patients need selective temporal lobectomy (or amygdalo-hippocampectomy) for long-term seizure control (6, 9,10). Amongst those patients in which seizures were initially controlled with antiepileptic drugs, there are patients who further become refractory to antiepileptic drugs and necessitate surgery (10). In a high percentage of patients, a silent period exists 
between the first seizure and the onset of intractability - this could indicate that the pathological substrate is progressive (3). $2 / 3$ of patients are seizure-free after 2-3 years after surgery for MTLE $(3,9)$. Recurrence of seizure in $1 / 3$ of patients is seen in incomplete resections of mesial temporal structures, in patients without evidence of histopathology in the surgical specimen or in those with incomplete resection, and it is a predictor of poor outcome (9). Recurrent seizures are associated with a history of secondarily generalized seizure $(3,9)$. Abnormalities outside the hippocampus (cortical malformations) are also associated with poor surgical prognosis $(9,10)$.

Over the time, MTLE was considered a single disorder. Now, it is viewed as a group of closely related syndromes with variable type and extent of histopathology (9).

\section{REFERENCES}

1. Thom M. Review: hippocampal sclerosis in epilepsy: a neuropathology review. Neuropathology and Applied Neurobiology. 2014; 40: 520-543.

2. Malmgren K., Thom M. Hippocampal sclerosis-Origins and imaging. Epilepsia. 2012; 53: 19-33.

3. ILAE Commission on Neurosurgery of Epilepsy. Mesial Temporal Lobe Epilepsy with Hippocampal Sclerosis. Epilepsia. 2004; 45: 695-714.

4. Tellez-Zenteno J.F., Hernandez-Ronquillo L. A review of the epidemiology of temporal lobe epilepsy. Epilepsy Research and Treatment. 2012.

5. Blair R.D.G. Temporal Lobe Epilepsy Semiology. Epilepsy Research and Treatment. 2012.

6. Baulac M. MTLE with hippocampal sclerosis in adult as a syndrome. Rev Neurol (Paris). 2015, 171: 259-66.

7. Tarkka R., Pääkkö E., Pyhtinen J. et-al. Febrile seizures and mesial temporal sclerosis: No association in a long-term follow-up study. Neurology. 2003; 60:215-218.

8. Aynacý F.M., Özdirim E., Saatçi I. et al. Clinical, electrophysiological and neuropsychological findings of twenty-two children with mesial temporal sclerosis. The Turkish Journal of Pediatrics. 2003, 45: 221-230.

9. Thom M., Mathern G., Cross H. Mesial temporal lobe epilepsy: how do we improve surgical outcome? Ann Neurol. 2010; 68: 424-434.

10. Ferreira Caboclo L., Neves R., Jardim A.P. et al. Surgical and postmortem pathology studies: contribution for the investigation of temporal lobe epilepsy. Arq Neuropsiquiatr. 2012; 70: 945-952.

\section{CONCLUSIONS}

MTLE with HS remains a controversial pathology, with a possible genetic predisposition, with existence of initial precipitating factors and of a progressive nature. MTLE has an unique onset, generalized seizures being rare. They probably represent secondarily generalized seizures and may correlate with the extent of the lesion. Even though the majority of cases described in the literature present unilateral mesial temporal sclerosis, bilateral lesions are frequently misdiagnosed, suggested by postmortem studies and by recurrence of seizure after surgery. Memory impairment is a neuropsychological comorbidity usually seen in these cases. Patients under antiepileptic treatment obtain seizure freedom, but recurrence of seizures in $2 / 3$ of these patients makes surgery the standard of care for them.

11. Sadler R.M. The syndrome of mesial temporal lobe epilepsy with hippocampal sclerosis: clinical features and differential diagnosis. Adv Neurol. 2006; 97: 27-37.

12. Barnett A.J., Park M.M., Pipitone J. et al. Functional and Structural Correlates of Memory in Patients with Mesial Temporal Lobe Epilepsy. Front Neurol. 2015; 6:103

13. O'Brien T.J., Kilpatrick C., Murrie V. et al. Temporal lobe epilepsy caused by mesial temporal sclerosis and temporal neocortical lesions A clinical and electroencephalographic study of 46 pathologically proven cases. Brain. 1996; 119: 2133-2141.

14. Avesani M., Giacopuzzi S., Bongiovanni L.G. EEG-fMRI Evaluation of Patients with Mesial Temporal Lobe Sclerosis. The Neuroradiology Journal. 2014; 27: 45-54.

15. Bronen R. MR of Mesial Temporal Sclerosis: How much is enough? AJNR. 1998;19: 15-18.

16. Mueller S.G., Laxer K.D., Scanlon C. et al. Different structural correlates for verbal memory impairment in temporal lobe epilepsy with and without mesial temporal lobe sclerosis. Hum Brain Mapp. 2012, 32: 489-99.

17. Fayed N., Olmos S., Morales H. et al. Physical Basis of Magnetic Resonance Spectroscopy and its Application to Central Nervous System Diseases. American Journal of Applied Sciences 2006; 3: $1836-1845$. 